\title{
Modelle Massenvernichtungswaffenfreier Zonen im Nahen- und Mittleren Osten und am Persischen Golf
}

\author{
Rolf Mützenich*
}

\begin{abstract}
The notion of a Middle East nuclear weapon free zone, or a weapons of mass destruction free zone, sounds like a utopian dream. Although the issue has been on international agendas for more than 30 years, it has yet even to approach realization. This article examines the development towards a nuclear weapon free zone in the Middle East, taking into account the special regional problems resulting from a possible realization of this. After that, there will be a discussion of the Gulf Cooperation Council's (GCC) suggestion of the creation of a weapons of mass destructions free zone in the Persian Gulf.
\end{abstract}

Keywords: Nuclear-weapons free zone, Middle East, Persian Gulf, regional arms control

\section{Einleitung}

„Die Idee einer kernwaffenfreien Zone im Nahen und Mittleren Osten wird Vielen wie eine Utopie erscheinen, über die es sich nicht nachzudenken lohnt. “1

$r$ ahlreiche Abhandlungen, die sich mit den Möglichkeiten zur Schaffung einer nuklear- oder massenvernichtungswaffenfreien Zone im Mittleren Osten beschäftigen, sprechen von einem utopischen Ziel. Gleichzeitig versuchen sie jedoch, die Haupthindernisse auf diesem Weg zu identifizieren und Lösungsperspektiven zu erarbeiten. Die Antriebsfeder für diese Lösungssuche dürfte sein, dass auch andere Regionen es geschafft haben, sich auf die Errichtung nuklearwaffenfreier Zonen zu einigen.

Das Versäumnis, in den vierziger und fünfziger Jahren des vergangenen Jahrhunderts Nuklearwaffen für gesetzwidrig zu erklären, führte dazu, dass manche Regierungen sich um Zwischenschritte bemühten. Infolgedessen wurden Initiativen gestartet, Stationierung, Test, Gebrauch und Entwicklung nuklearer Waffen in bestimmten Regionen zu verbieten. Zunächst wurden nur unbewohnte Gebiete wie die Antarktis oder der Weltraum zu nuklearwaffenfreien Zonen erklärt. Im Jahre 1967 folgte durch den Vertrag von Tlatelolco mit Lateinamerika und der Karibik erstmals auch eine bewohnte Region. Mittlerweile sind in Südostasien und im Südpazifik weitere Verträge über nuklearwaffenfreie Zonen in Kraft getreten. Entsprechende Verträge wurden auch für Afrika und Zentralasien unterzeichnet, sind aber bislang noch nicht in Kraft getreten.

Das Konzept einer nuklearwaffenfreien Zone im Mittleren Osten reicht zurück bis in das Jahr 1974, als der Iran mit Unterstützung Ägyptens der UN-Vollversammlung die Errichtung einer solchen Zone vorschlug. Bis zum Ausbruch des zweiten Golfkrieges gab es diesbezüglich jedoch keine entscheidenden Fortschritte. Im Zuge des Golfkrieges wurde das Konzept einer nuklearwaffenfreien Zone zunehmend vom Konzept einer Region, die frei von sämtlichen Massenvernichtungswaffen ist, abgelöst.

\footnotetext{
* Dr. Rolf Mützenich, MdB, Mitglied im Auswärtigen Ausschuss, nahost- und abrüstungspolitischer Sprecher der SPD-Bundestagsfraktion

1 Claudia Baumgart/Harald Müller, Eitler Traum oder erreichbares Ziel? Die Idee einer kernwaffenfreien Zone im Nahen Osten, HSFK-Report Nr. 10/2004, Frankfurt/Main, S. 1.
}

Dieser Beitrag untersucht die Entwicklungen bezüglich einer massenvernichtung-waffenfreien Zone im Mittleren Osten unter Berücksichtigung der besonderen Probleme, die sich in der Region auf dem Weg zur Realisierung ergeben. Anschließend wird ein Vorschlag des Golfkooperationsrates (GKR) zur Schaffung einer massenvernichtungswaffenfreien Zone am Persischen Golf vorgestellt, der nicht die gesamte Region, sondern nur die GKR-Staaten sowie Irak, Iran und Jemen mit einbezieht. Warum hat der GKR diese Initiative ins Leben gerufen? Was sind die Haupthindernisse auf dem Weg zur Errichtung einer solchen Zone? Wie sind die Erfolgsaussichten zu bewerten? Daran schließt sich ein Kapitel über Modelle für ein Regionales Sicherheitssystem an. Dieses folgt der Grundannahme, dass ohne ein institutionalisiertes Forum, das den sicherheitspolitischen Dialog fördert, die Erfolgsaussichten für eine Abrüstung aller Massenvernichtungswaffen in der Region sehr gering sind. ${ }^{2}$

\section{Absichten und Folgen regionaler Rüstungs- kontrollkonzepte ${ }^{3}$}

Atom- und massenvernichtungswaffenfreie Zonen sind Teil des rüstungskontrollpolitischen Instrumentariums. In erster Linie sind derartige Abkommen Nicht-Rüstungsverträge. Die Mitglieder verpflichten sich, auf die Herstellung, die Verfügung, den Erwerb und die Lagerung eines Waffensystems zu verzichten. Des Weiteren kann auch der Transport durch das Vertragsgebiet oder Teile davon untersagt werden. In diesem Fall erwarten sie in einem Zusatzprotokoll die Respektierung durch die Kernwaffenmächte. In der Regel sollen sich die Atommächte verpflichten, auf die Drohung mit und den Ersteinsatz von Kernwaffen zu verzichten und nicht gegen die Vertragsinhalte zu verstoßen. Nicht-Rüstungsverträge haben den Vorteil, dass sie die Rüstungskosten mindern, die Einsatzoptionen und den Schaden in einem militärischen Konflikt beschränken und Rüstungswettläufe im Vorhinein ausschließen. Sie können dazu beitragen, dass Sicherheitsdilemma zu durchbrechen.

2 Vgl. Peter Jones, A Gulf WMD Free Zone within a Broader Gulf and Middle East Security Architecture, Policy Papers (Gulf Research Center), 2005.

3 Vgl. Rolf Mützenich, Atomwaffenfreie Zonen und internationale Politik: historische Erfahrungen, Rahmenbedingungen, Perspektiven, Frankfurt am Main 1991. 
Indem sich Staaten in einem Abkommen zur Rüstungsbeschränkung zusammenfinden, fördern sie die regionale $\mathrm{Zu}$ sammenarbeit. In der Regel werden derartige Verträge durch Institutionen abgesichert, die mit Hilfe von Berichtspflichten, Überwachung und Konferenzen auf die Einhaltung der Verträge achten. So wird der Weg zu Vertrauensbildung und der Herausbildung regionaler Gemeinsamkeiten geebnet. In den Fällen der Kernwaffenfreien Zonen in Südostasien (Treaty of Bangkok) und in Afrika (Treaty of Pelindaba) waren die Abkommen „Türöffner" für ehemals Außenstehende. So war zwar der Treaty of Bangkok durch die Kerngruppe der südostasiatischen Staatengemeinschaft ASEAN entwickelt und unterzeichnet worden, doch Vietnam, Laos und Kambodscha traten dem Vertragswerk ebenfalls bei. Erst später wurden sie Mitglieder der ASEAN-Staatengruppe. Südafrikas Beitritt zur Afrikanischen Union gestaltete sich dank des Treaty of Pelindaba einfacher. Im Fall der kernwaffenfreien Zone im Südpazifik (Treaty of Rarotonga) etablierten sich die ehemals „weißen“ Länder Australien und Neuseeland als Teil der südpazifischen Inselwelt. Selbst dort, wo wie in Zentralasien multilaterale Foren der Zusammenarbeit noch in den Anfängen stecken und zwischenstaatliche Konflikte vorherrschen, wurde eine nuklearwaffenfreie Zone (Treaty of Semipalatinsk) geschaffen. ${ }^{4}$

Regionale Verträge sind Teil internationaler Regimebildung und verstärken die Tendenz norm- und regelgeleiteter Kooperation zwischen Staaten und Regionen. Sie enthalten Prinzipien, Normen, Regeln und Verfahren um Konflikte und Probleme zu bearbeiten. Kern- und Massenvernichtungswaffenfreie Zonen können der Friedenssicherung dienen und koordinieren ein gemeinsames Verhalten der Regimemitglieder gegenüber der Umwelt.

Dabei können Verträge zugunsten atom- und massenvernichtungswaffenfreier Zonen nicht nur die regionale Identität befördern, sondern auch zur regionalen Autonomisierung beitragen. Die Beschränkung der Handlungsoptionen außerregionaler Akteure ist offensichtlich. Der Treaty of Bangkok diente den Philippinen nicht nur dazu, den Preis für US-Marinebasen hochzuschrauben. Vielmehr erschwerte der Vertrag die Bewegungsfreiheit kernwaffenbestückter Schiffe und griff die umstrittene Ausdehnung der nationalen Grenzen durch die Seerechtskonvention auf. Sowohl die USA (Freiheit der Meere) als auch die VR China (Inseln im Südchinesischen Meer) fühlten sich durch die Vertragsgestaltung in ihren Ansprüchen herausgefordert. Auch das Abkommen im Südpazifik war in erster Linie geschaffen worden, um die Nutzung der Inseln für oberund unterirdische Atomwaffentests durch Frankreich und die USA zu behindern. Die kernwaffenfreie Zone in Lateinamerika und der Karibik (Treaty of Tlatelolco) war vor allem eine Reaktion auf die Kuba-Krise. Den USA und der Sowjetunion sollten Beschränkungen auferlegt und die Folgen des Ost-West-Konflikts abgeschwächt werden.

Massenvernichtungswaffenfreie Zonen sind keine Utopie. Sie sind machbar, wenn ein gemeinsames Interesse und ein ge-

4 Am 8. September 2006 unterzeichneten die Außenminister der fünf zentralasiatischen Staaten (Kasachstan, Kirgisistan, Tadschikistan, Turkmenistan und Usbekistan) in Semipalatinsk, dem ehemaligen Atomwaffentestgelände der Sowjetunion in Kasachstan, den Vertrag über eine Kernwaffenfreie Zone in Zentralasien. meinsamer politischer Wille vorliegen. Demnach sollte man auch dort nach Möglichkeiten Ausschau halten, wo scheinbar unüberbrückbare Unterschiede und Konflikte vorherrschen. Abgestufte Schritte könnten eine Vorstufe für weitere subregionale Rüstungskontrollregime sein. Für den Nahen und Mittleren Osten sind angesichts der dramatischen Folgen einer unbegrenzten Nuklearisierung der Region keine Denkverbote erlaubt.

\section{Massenvernichtungswaffenfreie Zone im Mittleren Osten}

Die Realisierung der Idee einer nuklearwaffenfreien Zone im Mittleren Osten lag bis zum Ausbruch des zweiten Golfkrieges in weiter Ferne. Angesichts eines fortdauernden militärischen Ungleichgewichts in der Region und der Frustration über fehlende Fortschritte hinsichtlich einer nuklearwaffenfreien Zone, brachte der ägyptische Präsident Hosni Mubarak eine Erweiterung des Konzepts hin zu einer massenvernichtungswaffenfreien Zone im Mittleren Osten (MEWMDFZ) ein. Die Gründe für diese Konzepterweiterung liegen zum einen im Iran-Irak-Krieg und zum anderen im zweiten Golfkrieg. Diese Kriege zeigten die Gefahren auf, die von einem Persischen Golf mit Massenvernichtungswaffen ausgehen. ${ }^{5}$ Schon 1991 hatte der UN-Sicherheitsrat in seiner Resolution 687 betont, dass die Abrüstung des Irak einen Schritt auf dem Weg zu einer Region, die frei von Massenvernichtungswaffen und Trägerraketen ist, darstellen sollte. ${ }^{6}$

Zur Lösung des israelisch-arabischen Konflikts und um die Sicherheit in der Region dauerhaft zu gewährleisten, wurde Ende 1991 der Madrid-Prozess in Gang gesetzt. Die Arms Control and Regional Security Working Group (ACRS) wurde eingesetzt, um Abrüstungsgespräche zwischen Israel und seinen arabischen Nachbarstaaten (mit Ausnahme Libyens und Irans) zu forcieren. Ein entsprechendes Abkommen kam aber nicht zustande, da Ägypten Israels Nuklearwaffenpotenzial sofort auf der Tagesordnung sehen wollte, während Israel dazu erst nach dem erfolgreichen Abschluss eines Rüstungskontrollabkommens bereit war. Diese Differenzen entsprangen aus sich diametral entgegengesetzten Wahrnehmungen des israelischen Nuklearwaffenpotenzials. Während man in Israel die Nuklearwaffen als Abschreckungsmaßnahme gegen feindlich gesinnte arabische Nachbarstaaten betrachtet, glaubt man in den arabischen Ländern an eine offensive Maßnahme, um die besetzten Gebiete zu erhalten, da die überlegene israelische Armee zur Selbstverteidigung stark genug sei. Verstärkt werden diese Wahrnehmungen durch extremistische Gruppen auf beiden Seiten. ${ }^{7}$

Trotz aller Herausforderungen und Hindernisse, die sich auf dem Weg zu einer massenvernichtungswaffenfreien Zone im Mittleren Osten ergeben, erscheint dieses Konzept zumindest erfolgversprechender als das einer Zone, die „lediglich“ von Nuklearwaffen dauerhaft befreit wird. Mehrere Länder des

5 Vgl. Gawdat Bahgat, Prospects for a Nuclear Weapons Free Zone in the Middle East, in: World Affairs 169 (4), 2007, S. 161-168, S. 164.

6 Vgl. United Nations, Security Council, Resolution 687 (1991), in: http://www fas.org/news/un/iraq/sres/sres0687.htm, (Zugriff: 01.04.2008).

7 Vgl. Claudia Baumgart, Harald Müller: A Nuclear Weapons-Free Zone in the Middle East: A Pie in the Sky? In: The Washington Quarterly 28 (1), 2004, S. $46-49$ (45). 
Mittleren Ostens besitzen chemische oder biologische Waffen. Andere haben die entsprechenden Verträge bezüglich eines Verbots dieser Waffen nicht unterzeichnet oder ratifiziert:

„presumed Israeli nuclear capability, and Iranian lack thereof, is considered a major reason for the proliferation of chemical and biological weapons in the region. Unable to achieve nuclear capability, Israel's adversaries have sought other WMDs as second-best deterrents."8

Eine Grundbedingung für die Abrüstung seines Nuklearwaffenpotenzials wäre für die israelische Regierung somit eine komplette Abrüstung von Massenvernichtungswaffen und ein Verbot dieser in der Region.

Eine weitere Bedingung für die nukleare Abrüstung sind erhebliche Fortschritte im israelisch-arabischen Friedensprozess. Sollten diese Fortschritte erzielt werden, könnten Abrüstungsabkommen geschlossen werden, die dann wiederum zu dauerhaftem Frieden führen. Hierbei ist jedoch zu bedenken, dass Israel Inspektionen seiner Atomanlagen nur zustimmen würde, wenn dies unter nationaler Aufsicht oder zumindest in gleichberechtigter Kooperation mit internationalen Inspektoren geschehen würde, da die israelische Regierung der Internationalen Atomenergiebehörde (IAEA) nur wenig Vertrauen entgegenbringt. Die arabischen Staaten hingegen möchten diese Aufgabe in die Hände der IAEA legen. Außerdem müsste gewährleistet sein, dass ein Bruch des Vertrages über eine massenvernichtungswaffenfreie Zone harte Konsequenzen nach sich zieht, sei es durch eine Garantiemacht oder durch den Sicherheitsrat. ${ }^{9}$

Das iranische Atomprogramm bildet einen weiteren Faktor, der die Realisierung einer massenvernichtungswaffenfreien Zone im Mittleren Osten erschwert, da ein neuer potenzieller $\mathrm{Nu}$ klearwaffenstaat auf den Plan getreten ist. Im September 2005 wurde festgestellt, dass der Iran sich nicht im Einklang mit den Safeguards des Nichtverbreitungsvertrags befindet, was einen Bericht an den UN-Sicherheitsrat nach sich zog. Die IAEAInspektoren stellten ferner fest, dass der Iran im Laufe von 18 Jahren zahlreiche Verletzungen der Safeguards begangen und sein Atomprogramm systematisch verschleiert hat. Hinzu kommen zahlreiche Indizien, die auf eine Verknüpfung des zivilen Atomprogramms mit militärischen Zwecken bis mindestens 2003 hinweisen. ${ }^{10}$ Auch jüngste Entwicklungen, einschließlich der IAEA-Berichte an den Sicherheitsrat, legen die Vermutung nahe, dass Irans Atomprogramm nicht ausschließlich zivilen Zwecken dient:

„Indeed, Iran's insistence, in the face of strong international opposition, on going ahead with uranium enrichment as well as the research reactor at Arak, ideal for plutonium production, suggests that it is moving to put in place the wherewithal for a weapons capability. “11

Für die Realisierung einer massenvernichtungswaffenfreien Zone im gesamten Mittleren Osten stellt das iranische Atom-

8 Bahgat, Prospects for a Nuclear Weapons Free Zone in the Middle East, S. 167.

9 Vgl. Baumgart/ Müller: A Nuclear Weapons-Free Zone in the Middle East: A Pie in the Sky? S. 51-55.

10 Mark Fitzpatrick, Is Iran's Nuclear Program Intended Solely For Civilian Purposes? In: Security \& Terrorism 7 (Gulf Research Center), 2007, S. 18-21.

11 Ebd., S.21. programm ein erhebliches Hindernis dar, weil es Anreize für die Staaten am Persischen Golf schafft, ebenfalls nuklear aufzurüsten und somit ein Gegengewicht zu einer potenziellen nuklearen Hegemonialmacht zu schaffen.

Im Dezember 2006 kündigte der Generalsekretär des Golfkooperationsrates (GKR) folgerichtig an, dass die sechs Mitglieder ${ }^{12}$ beabsichtigen, ein gemeinsames nukleares Forschungsprogramm in Gang zu setzen. Zusätzlich kündigten die Vereinigten Arabischen Emirate, Bahrain und Kuwait die Verfolgung eines separaten, nationalen Nuklearprogramms in Zusammenarbeit mit der IAEA an. Die Ankündigung kam überraschend, da diese Staaten bisher die Politik einer "zero nuclear option“ verfolgten. Außerdem galt bisher, dass Opposition gegen das iranische Atomprogramm nicht offen betrieben wird, solange Israels Nuklearwaffenpotenzial von einem „double standard“ in der Atomfrage zeugt: „as long as no action is taken against Israel, opposition to Iranian nuclear programs is something of a taboo in the Muslim world."13 Die Motivation für diese Atomprogramme entspringt neben Aspekten wie der Diversifizierung der Energieversorgung, der Schließung der „nuclear technological gap“ oder umweltschonender Energieerzeugung vor allem dem Wunsch, Iran zu signalisieren, dass die Staaten des Persischen Golfs sich durch das iranische Atomprogramm bedroht fühlen: „The joint nuclear research program is also a subtle signal to Iran that the GCC is leaving its options open to develop a military program." 14

Die Realisierung einer massenvernichtungswaffenfreien Zone für den gesamten Mittleren Osten scheitert, zusammengefasst, bisher vor allem an zwei Polen. Zum einen sind bis heute keine durchschlagenden Erfolge bezüglich israelisch-arabischer Abrüstungsinitiativen erzielt worden. Zum anderen hat das iranische Atomprogramm, das vor allem wegen der Verschleierungstaktik und der Dimension zur begründeten Annahme eines militärischen Zweckes Anlass gibt, zu einer erheblichen Verschlechterung der Beziehungen zwischen dem Iran und den Golfanrainern geführt.

Weniger ehrgeizige Ziele im Sinne eines „step-by-step approach“ zur Schaffung kleinerer massenvernichtungswaffenfreier Zonen, die eine magnetische Wirkung auf Nachbarstaaten ausüben, könnten das Spannungsfeld dieser Pole durchbrechen:

„Comme l'incitation à acquérir des armes nucléaires peut provenir des considérations régionales ou sous-régionales, les pays assurés que leurs voisins ne possédent pas d'armes nucléaires peuvent être moins enclins à acquérir de telles armes eux-mêmes. Ce raisonnement s'applique à toutes les catégories d'armes de destruction massive."15

12 Bahrain, Oman, Kuwait, Saudi-Arabien, Katar, Vereinigte Arabische Emirate.

13 Emily B. Landau, Taking a Stand On Nuclear Iran: Voices From The Persian Gulf, in: Tel Aviv Notes 157, 2006, S. 1-3, S. 1.

14 Nicole Stracke, Nuclear Development in the Gulf: A Strategic or Economic Necessity? In: Security \& Terrorism 7 (Gulf Research Center), 2007, S. 4-10, S. 4-7 (6).

15 Übersetzung des Autors: „Da der Anreiz zur atomaren Bewaffnung seinen Ursprung in regionalen oder sub-regionalen Erwägungen haben kann, können im Umkehrschluss die Länder, die sich sicher sein können, dass ihre Nachbarn keine atomaren Waffen besitzen, weniger geneigt sein, sich selbst atomar zu bewaffnen. Diese Logik lässt sich auf alle Bereiche von Massenvernichtungswaffen anwenden." Jozef Goldblat, En quoi le projet GWMDFZ est-il important? In: Bulletin d'information de l'Institut International de Recherches pour la Paix à Genève (Fondation GIPRI), 2006, S.6-7, (6). 
Wichtiger Ansatzpunkt in diesem Zusammenhang ist der Treaty of Pelindaba über eine afrikanische nuklearwaffenfreie Zone, ${ }^{16}$ der 1996 in Kairo unterzeichnet, aber noch nicht von allen Staaten ratifiziert wurde. Unter den Unterzeichnern dieses Vertrages finden sich einige Länder, die auch zu einer massenvernichtungswaffenfreien Zone des gesamten Mittleren Ostens gehören würden, wie beispielsweise Ägypten.

"The denuclearisation of the African part of the prospective NWFZME could be considered the first step in a step-by-step approach to the zone-building. An interesting initiative along the same line is the proposal to establish a weapons of mass destruction free zone in the Gulf area (GWMDFZ) being researched by the Gulf Research Center in Dubai, UAE."17

\section{Massenvernichtungswaffenfreie Zone am Per- sischen Golf}

Im Jahre 2004 wurde durch das Gulf Research Center (GRC) in Dubai die Idee einer massenvernichtungswaffenfreien Zone am Persischen Golf (GWMDFZ) eingeführt. Nach mehreren sogenannten Track-II-Runden, in denen Wissenschaftler, Militärs und Diplomaten zusammenkamen, um fernab von offiziellen, diplomatischen Verhandlungen zu diskutieren, hatte das Projekt derart positive Resonanz erhalten, dass der Generalsekretär des Golfkooperationsrates (GKR) im Dezember 2005 die Initiative des GKR zur Schaffung einer GWMDFZ ankündigte. Diese Zone sollte die sechs Mitgliedstaaten des Rates sowie Iran, Irak und Jemen umfassen. Die Idee einer GWMDFZ basiert auf dem „sub-regional to regional approach“, beginnend mit Sicherheitskooperation unter den neun Golf-Staaten und einer möglichen Öffnung für eine Expansion auf den gesamten Mittleren Osten inklusive Israels. Die subregionale Kooperation der Golfstaaten entspringt der gemeinsamen Wahrnehmung, dass die Bedrohungslage sich anders darstellt als bei anderen arabischen Staaten. Vorrangig ist in diesem Zusammenhang neben der Lage im Irak der regionale Konflikt mit Iran zu nennen: „While the GCC states, which are geographically close to Iran, would perceive it as a direct threat, Egypt or Morocco views Iran's threat as less imminent. Therefore, the GCC states feel that security cooperation is more effective among states that share common interests and threat perceptions. "18

Gegenwind bekam die GKR-Initiative von der Arabischen Liga. Deren Generalsekretär Amr Moussa sieht darin eine Gefahr für eine zu schaffende MEWMDFZ, die Israel mit einbezieht. Amr Moussa äußert die Befürchtung, dass Israel vom politischen Druck befreit werden könnte, der sich aus dem Prinzip der Schaffung einer massenvernichtungswaffenfreien Zone im Mittleren Osten ergibt. ${ }^{19}$

16 http://www.fas.org/nuke/control/anwfz/text/afrinwfz.htm (Zugriff: 02.04.2008).

17 Jan Prawitz, A Note on the Proposed Zone Free of Weapons of Mass Destruction in the Middle East, in: http://www.cisd.soas.ac.uk/Editor/assets/wmdfz me\%20\%prawitz\%20070917.pdf (Zugriff: 27.03.2008).

18 Vgl. Gulf Research Center, The Nuclear/WMD Free Zone in the Gulf: A Track II Initiative, in: Security \& Terrorism 7 (Gulf Research Center), 2007, S.31.

19 Amr Mousa (Secretary General of the Arab League), The Debate with the Arab League on the Gulf as a WMDFZ. The Letter of the Secretary-General of the Arab League to the Secretary-General of the GCC, in: Security \& Terrorism 7 (Gulf Research Center), 2007, S. 33-34
Auch der Iran hat sich gegen eine solche Initiative, die keinerlei Vorbedingungen an Israel stellt, ausgesprochen. Stattdessen haben iranische Diplomaten in Track-II-Verhandlungen die iranische Vorbedingung deutlich gemacht: „During the ongoing Track II negotiations, Iranian officials made the nuclear disarmament of Israel a pre-condition for Irans's membership in the Gulf-WMDFZ knowing fully that such a pre-condition would lead to a deadlock. “20

Der Golfkooperationsrat hat sich jedoch eindeutig für das zunächst bescheidenere Ziel einer massenvernichtungswaffenfreien Zone am Persischen Golf entschieden. Track-II-Dialoge, die vor allem im Laufe der 1990er Jahre viele Experten, Militärs und Diplomaten des Nahen und Mittleren Ostens zu inoffiziellen sicherheitspolitischen Gesprächen zusammengeführt haben, gestalteten sich in den letzten Jahren schwieriger. Die Gründe hierfür sind vor allem in fehlenden Fortschritten bei der Lösung des israelisch-palästinensischen Konflikts zu suchen sowie in der allgemein angespannten Lage im Mittleren Osten, die eine arabisch-israelische Kooperation erheblich verkompliziert. Außerdem konnten Ergebnisse inoffizieller arabisch-israelischer Gespräche selten an die Öffentlichkeit gebracht und in offizielle Politik transferiert werden „Domestic environments make participants cautious about exposing track two ideas to wider audiences. Cooperation is still a dangerous position in the region, and Israelis are suspicious of cooperative postures that signal weakness. “21 In den letzten Jahren haben daher Ideen neuer regionaler Sicherheitssysteme an Beliebtheit gewonnen, deren Ursprung häufig in den Ländern am Persischen Golf zu finden ist. Auf diese Weise soll der sicherheitspolitische Dialog aus seiner Lethargie befreit werden.

Das Konzept einer massenvernichtungswaffenfreien Zone, die sich statt über den gesamten Mittleren Osten vorerst nur über die Länder am Persischen Golf erstreckt, mag auf den ersten Blick relativ einfach zu realisieren erscheinen. Die Gründe, die Iran dazu veranlassen, mit seinem Atomprogramm weiterhin den eingeschlagenen Kurs beizubehalten, deuten jedoch auf das Gegenteil hin. Erstens hat der Sturz Saddam Husseins Iran zwar von einer direkten Bedrohung durch den Irak befreit, aber die Tatsache, dass die Vereinigten Staaten in einem Nachbarland mit einer großen Armee präsent sind, beunruhigt die Teheraner Regierung. Zweitens bleibt Israel, wenn auch kein akutes Sicherheitsproblem, so doch zumindest ein ideologischer Gegner. Drittens hat der Gebrauch von Chemiewaffen gegen den Iran im ersten Golfkrieg sowie die fehlende internationale Reaktion auf diesen Bruch des Genfer Protokolls von $1925 \mathrm{zu}$ Misstrauen gegenüber internationalen Rüstungsabkommen geführt. Viertens trägt die regionale Unsicherheit, inklusive des nuklearen Status' der Nachbarn Pakistan und Indien, zu einem Unsicherheitsgefühl bei. Schließlich führen der regionale Machtanspruch und ein Gefühl der Diskriminierung dazu, dass der Iran sich die Möglichkeit einer zukünftigen Aufrüstung mit

20 Nicole Stracke, Nuclear arms race in the Gulf, in: Khaleej Times Online, 02.02.2007.

21 Vgl. Dalia Dasse Kaye (RAND Corporation), Talking to the Enemy. Track Two Diplomacy in the Middle East and South Asia, Santa Monica/ Arlington/ Pittsburgh 2007, S. xiv-xv (xv). 
Massenvernichtungswaffen offenhalten will. ${ }^{22}$ Das Konzept einer GWMDFZ erscheint trotz dieser Hindernisse im Hinblick auf den Iran lohnenswert:

„providing it can indeed be achieved without diminishing the importance of the wider call for a zone including the whole of the Middle East including Israel. In the interim it could provide valuable security benefits for the nine states proposed as members. Its precise content must be matter for those states to negotiate, taking due account of the concerns of their neighbours and the wider community."23

Zum gegenwärtigen Zeitpunkt erscheint es unwahrscheinlich, dass der Iran sich bedingungslosen Verhandlungen über eine massenvernichtungswaffenfreie Zone am Persischen Golf anschließt. Gleichwohl könnten Verhandlungen über ein derartiges Vertragswerk helfen, gegenseitiges Misstrauen im regionalen Umfeld abzubauen, die Aussichten für einen regionalen Abrüstungsdialog zu verbessern und eventuell neue Impulse für ein kooperatives regionales Sicherheitssystem im gesamten Mittleren Osten freizusetzen, das sowohl Iran als auch Israel umfasst. $^{24}$

Die Initiative des Golfkooperationsrates kann einen entscheidenden Beitrag für ernsthafte Diskussionen über eine WMDFZ für den gesamten Mittleren Osten leisten. Die Erfahrungen in anderen massenvernichtungswaffenfreien Zonen haben gezeigt, dass die Initiierung eines solchen Prozesses von einigen Staaten ausging, die die Führung übernommen haben und denen sich später weitere Staaten angeschlossen haben, sobald es die politischen Realitäten erlaubt haben. Eben diese Erfahrungen anderer WMDFZs haben aber auch deutlich gemacht, dass keine massenvernichtungswaffenfreie Zone etabliert wurde, ohne deren Realisierung in ein umfassenderes regionales System für Kooperation und Dialog einzubetten. Solche Systeme schaffen ein weithin akzeptiertes Gerüst an regionalen Verhaltens- und Verfahrensnormen, durch welche Nachbarstaaten ihre Unstimmigkeiten friedlich lösen können. ${ }^{25}$ Für eine massenvernichtungswaffenfreie Zone am Persischen Golf oder im Mittleren Osten erscheint ein solches System ebenfalls unerlässlich: „It is very hard to believe the Middle East, or the Gulf, will be able to create a WMDFZ without also, and probably first, taking considerable steps to develop a broader regional security and cooperation architecture. "26 Im Folgenden sollen zwei Modelle eines solchen Sicherheitssystems für den Mittleren Osten vorgestellt werden.

22 Vgl. Jones, A Gulf WMD Free Zone within a Broader Gulf and Middle East Security Architecture, S. 8.Policy Papers (Gulf Research Center), März 2005, S. 8 .

23 Ian R. Kenyon, The project for a Gulf Zone Free of Weapons of Mass Destruction, S. 4, in: http://www.ippnw.ch/content/pdf/monte_veritas/I_Kenyon. pdf (Zugriff:29.03.2008).

24 Vgl. Landau, Taking a Stand On Nuclear Iran: Voices From The Persian Gulf, S. $2-3$.

25 Vgl. Jones, A Gulf WMD Free Zone within a Broader Gulf and Middle East Security Architecture, S. 5-12.

26 Ebd., S. 12.

\section{Regionales Sicherheitssystem für den Mittle- ren Osten}

Seitdem Großbritannien 1968 seinen Rückzug aus dem Gebiet östlich des Suez-Kanals angekündigt hatte, gab es wiederholte Bemühungen zur Schaffung eines effektiven Sicherheitssystems am Persischen Golf. Bis heute jedoch wurde kein Sicherheitssystem in der Region institutionalisiert. Stattdessen basiert die militärische Sicherheit in der Region, nach dem Rückzug Großbritanniens, seit den 1970er Jahren auf den Vereinigten Staaten. Diese haben bisher regionale Verbündete unterstützt, um das Machtungleichgewicht auszubalancieren. Die USA unterhalten ferner diverse Militärstützpunkte in der Region am Persischen Golf. Frankreich verfolgt ähnliche Pläne.

In anderen Regionen der Welt, wie z.B. in Europa, Ostasien und Südamerika, wurde nach Ende des Kalten Krieges die traditionelle, realpolitische Perspektive internationaler Beziehungen, die durch ein Nullsummen-Denken bezüglich nationaler Sicherheit gekennzeichnet ist, abgelöst. Stattdessen wurde der Sicherheitsbegriff um Fragen der politischen Legitimität und ökonomische Aspekte erweitert, so dass zusätzlicher Spielraum für die Zusammenarbeit in der Sicherheitspolitik geschaffen wurde. Diese Dynamik trifft auf die Region am Persischen Golf jedoch nicht zu. Bis heute verlassen sich die USA zur Wahrung der Sicherheit vor allem auf Gegenmachtbildung, da die Erfahrungen des Iran-Irak-Krieges und des Einmarschs Iraks in Kuwait deutlich machten, dass eine Verschiebung des Mächtegleichgewichts zu Krieg führen kann. ${ }^{27}$

Die US-amerikanische Politik der Gegenmachtbildung hat sich im letzten Jahr durch angekündigte umfangreiche Waffenlieferungen vor allem an Ägypten, Israel und Saudi-Arabien, aber auch an die Staaten des Golfkooperationsrates, verdeutlicht. Das Ziel der amerikanischen Administration ist es, auf diese Weise den regionalen, iranischen Hegemoniebestrebungen entgegenzuwirken.

Die Tatsache, dass die bisherige Politik zu keinem dauerhaften Frieden am Persischen Golf geführt hat, der mangelnde institutionelle Rahmen zum Umgang mit der bestehenden Machtasymmetrie am Golf sowie das Dilemma der Golfstaaten, dass ihre Sicherheit zwar vom Schutz der Vereinigten Staaten abhängt, ihre Bevölkerungen die amerikanische Präsenz aber zum überwiegenden Teil ablehnen, führt konsequenterweise zu Überlegungen einer geeigneten regionalen Sicherheitsarchitektur. ${ }^{28}$

Ein häufig artikuliertes Modell eines regionalen Sicherheitssystems ist eine Konferenz für Sicherheit und Zusammenarbeit im Mittleren und Nahen Osten (KSZMNO), die sich an der Konferenz über Sicherheit und Zusammenarbeit in Europa (KSZE) orientiert. Auf diese Weise sollen alle Einzelkonflikte der Region in „einen für die Lösungsperspektive adäquaten politischen Kontext gestellt werden“. ${ }^{29}$ Ausgangspunkt für Überlegungen zur Schaffung einer KSZMNO ist somit die Erkenntnis, dass die

27 Vgl. Andrew Rathmell/ Theodore Karasik et al., A New Persian Gulf Security System, Issue Paper 248 (RAND), 2003, S. 1-3.

28 Vgl. Katja Niethammer, Vertrackte Golf-Spiele. Die Staaten des Golfkooperationsrates und der Iran, SWP-Aktuell 26, 2006, S. 3-4.

29 Mohssen Massarat, Friedensmacht Europa?, in: http://www.gcn.de/download/Friedensmacht Europa.pdf, S. 3 (Zugriff: 30.03.2008). 
zahlreichen grenzüberschreitenden Konflikte in der Region wie z.B. der Israel-Palästina-Konflikt, der Kurdistan-Konflikt und Konflikte bezüglich der Nutzung von Gewässern und Ölquellen nur in einem kooperativ angelegten regionalen Ordnungsrahmen gelöst werden können. Grundvoraussetzung für eine derartige Konferenz ist die Bereitschaft Israels, die Perspektive einer Abrüstung seines Potenzials an Massenvernichtungswaffen anzubieten sowie umgekehrt eine Sicherheitskooperation der arabischen Staaten mit Israel, die jedoch vom Sicherheitsrat der Vereinten Nationen mit einer Sicherheitsgarantie für Israel unterfüttert werden müsste. ${ }^{30}$

Da der Sicherheitsbegriff seit Ende des Kalten Krieges eine Erweiterung erfahren und sich die Erkenntnis durchgesetzt hat, dass Sicherheit sich neben der militärischen auch auf die wirtschaftliche, ökologische oder die Energieebene erstreckt, muss eine Sicherheitskonferenz und eine sich daraus entwickelnde Organisation für Sicherheit und Zusammenarbeit im Mittleren und Nahen Osten all diese Themenfelder berücksichtigen. Die Schaffung einer massenvernichtungswaffenfreien Zone kann somit nicht isoliert betrachtet werden, jedoch als erster wichtiger Anhaltspunkt für eine Neuordnung der gesamten Region dienen.

Ein anderes Modell eines effizienten Sicherheitssystems konzentriert sich auf die Region am Persischen Golf - also zunächst ohne Israel einzubeziehen - und lehnt sich an die Südostasiatische Staatengemeinschaft (ASEAN) an. Grundlegend für das Plädoyer zugunsten des ASEAN-Modells sind diverse Gemeinsamkeiten zwischen den ASEAN-Staaten und den Golfstaaten:

- Asiatische Staaten, wie heute noch jene am Persischen Golf, waren während der regionalen Regimebildung keine Demokratien nach westlichem Vorbild. Einige Staaten der ASEANGemeinschaft sind dies bis heute nicht.

- Sie weisen einen ähnlich geringen intraregionalen und symmetrischen ökonomischen Austausch auf.

- Beide Regionen sind sehr konfliktanfällig. In beiden Regionen existieren Grenz- und Machtkonflikte.

- Das asiatische Sicherheitssystem gleicht jenem am Persischen Golf darin, dass in beiden Systemen regionale Mächte großen Einfluss ausüben und starke bilaterale Beziehungen zu den USA vorherrschen.

- Die ASEAN ist durch eine informelle, unbürokratische Entscheidungsfindung mit großem Respekt vor nationalstaatlicher Souveränität gekennzeichnet, was der politischen Kultur der Golfstaaten entgegenkäme. ${ }^{31}$

Den südostasiatischen Staaten ist es mit der Errichtung des ASEAN Regional Forum (ARF), an dem sich neben den ASEANMitgliedern zahlreiche externe Akteure wie die USA, die EU, Russland und China beteiligen, gelungen, ein breit angelegtes Forum für Sicherheitsdialoge zu etablieren. Hinzu kommt mit ASEAN + 3 (APT) eine straffere Organisation mit den ASEANMitgliedern sowie China, Japan und Südkorea, welche die Machtrealitäten in der Region besser widerspiegelt und für die Zukunft der Sicherheitskooperation in Südostasien verhei-

30 Vgl. ebd, S. 4-6.

31 Vgl. Cliff Kupchan, Toward a New Persian Gulf Security System: Lessons of the Asian Model, in: The Iranian Journal of International Affairs 19 (2), 2007, S.65-80 (65-68). ßungsvoll ist. ${ }^{32}$ Aufgrund der positiven Erfahrungen in Südostasien mit einem regionalen Sicherheitssystem und der Tatsache, dass diese Region eine nuklearwaffenfreie Zone errichten konnte sowie den Gemeinsamkeiten in Südostasien und am Persischen Golf bezüglich der Ausgangsbasis für Sicherheitskooperation, sollte sich ein Sicherheitssystem am Persischen Golf an der Ausgestaltung der ASEAN orientieren und folgende Grundsätze aufweisen:

- Nichteinmischung in nationale Angelegenheiten

- Regionale Lösungen für regionale Probleme

- Vermeidung von militärischen Pakten, da dies ein zu ehrgeiziges Ziel wäre

- Verzicht auf Gewaltanwendung und Verpflichtung zur friedlichen Beilegung von Konflikten

- Ein Mechanismus, der Konsensfindung durch intensive, hochrangige Beratungen unter Vermeidung von zu viel Bürokratie. ${ }^{33}$

Der Erfolg der südostasiatischen Staaten, mit diesem Modell eine nuklearwaffenfreie Zone zu errichten, gibt Anlass zur Hoffnung, dass dies auch am Persischen Golf oder auf mittelbis langfristige Sicht im gesamten Mittleren Osten gelingen kann, zumal sich die sicherheitspolitischen Grundstrukturen in beiden Regionen sehr ähneln. Demnach wäre die Unterzeichnung der Zusatzprotokolle des Treaty of Bangkok durch die fünf Atomwaffenmächte nicht allein ein wichtiges Signal an die ASEAN-Gemeinschaft. Ein solcher Schritt könnte auch im Persischen Golf Beachtung finden.

\section{Ausblick}

Die Erfahrungen bei der Errichtung nuklearwaffenfreier Zonen haben gezeigt, dass es sehr schwierig ist, eine solche Zone zu etablieren, wenn ein Staat in der Region - wie Israel im Mittleren Osten - bereits Nuklearwaffen besitzt. Der Vertrag von Pelindaba über eine afrikanische nuklearwaffenfreie Zone bildet die einzige Ausnahme. Südafrika hat seine Nuklearwaffen abgebaut und den Nichtverbreitungsvertrag unterzeichnet. Dieser Vorgang stellt jedoch eine einzigartige Ausnahme in der Geschichte der nuklearen Nichtverbreitung dar. ${ }^{34}$

Für die Region des Mittleren Ostens kommt erschwerend hinzu, dass es eine äußerst konfliktträchtige Region ist, in der mehrere Länder Massenvernichtungswaffen besitzen, kein dauerhafter Frieden zwischen den Ländern der Region geschlossen wurde und gegenseitiges Misstrauen vorherrscht. Über allem schwebt die Frage, was zuerst kommen sollte: Frieden oder Abrüstung. Während Israel einer Abrüstung seiner Nuklearwaffen nur bei Abschluss von robusten Friedenverträgen mit all seinen Nachbarn zustimmen würde, machen diese die Abrüstung zur Vorbedingung von Friedensverträgen.

Die Bemühungen zur Errichtung einer massenvernichtungswaffenfreien Zone im Nahen und Mittleren Osten seit den

32 Vgl. ebd., S.70-73.

33 Vgl. Kupchan, Toward a New Persian Gulf Security System: Lessons of the Asian Model, S. 73-80.

34 Vgl. Bahgat, Prospects for a Nuclear Weapons Free Zone in the Middle East, S. 167. 
frühen 1990er Jahren sind ernüchternd. Sie wiesen jedoch insofern in die richtige Richtung, als dass sie das Konzept einer nuklearwaffenfreien Zone auf alle Massenvernichtungswaffen in der Region erweiterten und somit die Wahrscheinlichkeit erhöhten, dass Israel sich eines Tages einer solchen Zone anschließen könnte. Zwar forderte Massarrat noch 2005, dass die EU sich kraft ihrer politischen und moralischen Macht für eine nuklearwaffenfreie Zone im Mittleren Osten einsetzen solle, um das Tor zum Frieden weiter aufzustoßen. ${ }^{35}$ Eine Grundbedingung für die Abrüstung seiner Nuklearwaffen ist für Israel jedoch die Abrüstung aller Massenvernichtungswaffen in der Region, weshalb die Forderung nach einer nuklearwaffenfreien Zone zu kurz gegriffen ist.

Angesichts der Tatsache, dass das erweiterte Konzept weit von seiner Realisierung entfernt ist, verdient die Initiative des GKR aus dem Jahre 2005 zur Schaffung einer massenvernichtungs-

35 Mohssen Massarrat, Irans Atomkonflikt in der Sackgasse: Neuer Golfkrieg kann nur durch Atomwaffenfreie Zone gebannt werden, in: http://www.bits. de/public/gast/massarrat4.htm (Zugriff: 15.04.2008). waffenfreien Zone am Persischen Golf besondere Beachtung. Dieses Konzept entspringt der Wahrnehmung, dass die Golfanrainerstaaten anderen Bedrohungen ausgesetzt sind als andere Länder der Region und eine Kooperation daher eher Aussicht auf Erfolg haben könnte. Trotz des Hindernisses, dass der Iran sich nur schwer in dieses Konzept einfügen wird, ist die Initiative des GKR lohnenswert, weil dessen Realisierung eine hohe Anziehungskraft auf Nachbarstaaten entfalten könnte, wie Erfahrungen mit den existierenden nuklearwaffenfreien Zonen gezeigt haben.

Alle Bemühungen um eine Abrüstung von Massenvernichtungswaffen im Mittleren Osten müssen jedoch in eine breit angelegte und institutionalisierte Sicherheitskooperation, die mittels vertrauensbildender Maßnahmen den Dialog fördert, eingebettet werden. Das südostasiatische Modell der ASEAN, die es unter einigermaßen vergleichbaren Umständen geschafft hat, eine nuklearwaffenfreie Zone zu errichten, kann hierbei am ehesten als Vorbild dienen.

\title{
Perspectives on War
}

\section{Collecting, Comparing and Disaggregating Data on Violent Conflicts}

\author{
Sven Chojnacki* and Gregor Reisch**
}

\begin{abstract}
Generating data is analytically crucial for the identification of empirical trends and for theoretically explaining the occurrence, escalation and duration of war. Practically, it contributes to the objective of developing preventive measures. In order to evaluate the evolution and transformation of war, in this article, we first examine the macro-trends for the period between 1946 and 2007 compiled in a new Consolidated List of Wars. In the second part, we compare data from major data-gathering projects to assess both their degree of convergence and their usefulness for the scientific study of war. In the final section we open the black box of war. We present disaggregated violent events for the case of Somalia which is a striking example of organized violence between mostly non-state armed groups, the collapse of state authority and variations of violent events in time and space.
\end{abstract}

Keywords: war, armed conflicts, violent events, data projects, quantitative conflict research

\section{Introduction}

The scientific study of war has come a long way. Incremental progress has been achieved with several islands of empirical findings and theoretical explanations. Today, we can build both on empirical support for specific propositions (e.g. democratic peace) and on productive theoretical debates (see, for example, the controversy over greed and grievance in civil wars or the growing incorporation of gender and civilian agency). Besides this good news of both additive and - at least to some degree - integrative accumulation of knowledge, a number of practical and analytical issues remain controversial or unresolved.

First and foremost, war as a social institution and a mechanism for the allocation of certain values is still a present feature of

\footnotetext{
* Dr. Sven Chojnacki is Professor of Peace and Security Studies at Freie Universität Berlin and Project Coordinator at the research center SFB 700 Governance in Areas of Limited Statehood, which is funded by the German Research Foundation.

** Gregor Reisch is Student Research Assistant at the research center SFB 700
} Governance in Areas of Limited Statehood. politics. It thus remains of central importance for the study of politics and social processes in general and conflict escalation in particular. While intra-state violence is the dominant form of war in the contemporary international system, it would be premature to regard inter-state wars as on the brink of extinction. The enduring rivalry between India and Pakistan or the recent war in the Caucasus between Russia and Georgia in August 2008, as well as the interventionism of some democracies in Kosovo, Afghanistan and Iraq raise some doubt about this hypothesis.

Secondly, in contradiction to the underlying logic of intersubjectivity the "world of war is what researchers make of it" (Eberwein/Chojnacki 2001: 29). The major data gathering projects such as the Correlates of War project (COW), the Uppsala Conflict Data Program (UCDP) or the Arbeitsgemeinschaft Kriegsursachenforschung (AKUF) at the University of Hamburg portray different worlds of wars depending on different definitions, operational criteria and classifications. One price to be paid for that pluralism is the problem of credibility in regard to con- 